\title{
Multimodality Cardiovascular Molecular Imaging: An Overview
}

\author{
Albert J. Sinusas \\ Departments of Medicine and Diagnostic Radiology, Yale University School of Medicine, New Haven, Connecticut
}

\begin{abstract}
A large, multidisciplinary symposium held at the National Institutes of Health in April and May 2009 brought together individuals from a wide range of scientific disciplines with the goal of promoting the nascent field of multimodality cardiovascular molecular imaging. This supplement to The Journal of Nuclear Medicine summarizes the presentations and discussions of that landmark symposium. The review articles not only present the state of the art of cardiovascular molecular imaging but also discuss opportunities and challenges the community will face in advancing cardiovascular molecular imaging to clinical practice. The use of molecular targets for imaging in preclinical models and the translation of these approaches to patients have become possible with the advent of several technologic advances reviewed in this supplement.
\end{abstract}

Key Words: molecular imaging; multimodality cardiovascular molecular imaging

J Nucl Med 2010; 51:1S-2S

DOI: 10.2967/jnumed.109.068122

M olecular imaging is emerging as a new approach for the noninvasive detection of molecular and cellular processes that can identify disease before the manifestation of gross anatomic features or physiologic consequences. Application of molecular imaging for early detection of the initiating events associated with disease will be critical for improved understanding of the underlying mechanisms of disease, primary prevention of disease, risk stratification of patients with disease, and promotion of individualized medical treatment based on the unique characteristics of a disease in any given patient. Current strategies involve the detection of targeted markers unique to the biologic process under investigation. The development of these biologically targeted markers has become possible with recent advances in molecular biology, including genomics and proteomics. The application of molecular imaging in the preclinical environment and subsequent translation to patient care have required the development of new high-sensitivity and high-resolution imaging technologies. The advancement and translation of molecular imaging requires the integration of multiple medical disciplines at both basic and clinical levels. There is a need for improved

Received Mar. 29, 2010; revision accepted Mar. 30, 2010.

For correspondence or reprints contact: Albert J. Sinusas, Yale University School of Medicine, Section of Cardiovascular Medicine, 333

Cedar St., 3FMP, P.O. Box 208017, New Haven, CT 06520-8017.

E-mail: albert.sinusas@yale.edu

COPYRIGHT () 2010 by the Society of Nuclear Medicine, Inc. communication between these critical basic scientists who discover new genes and cellular pathways, any of which could serve as diagnostic or therapeutic agents, and the imaging scientists and clinicians who could transform these discoveries into noninvasive imaging techniques for the diagnosis and treatment of disease. The advancement and translation of molecular imaging in relationship to detection and management of cardiovascular disease have been suboptimal.

\section{ADVANCEMENT OF CARDIOVASCULAR MOLECULAR IMAGING}

To promote improved management of patients with cardiovascular disease, there needs to be better collaboration between basic scientists, imaging scientists, and clinicians in order to advance the field of cardiovascular molecular imaging. The creation of multidisciplinary basic and translational research teams that use cellular and molecular imaging will lead to improved management of cardiovascular disease. The American Society of Nuclear Cardiology initiated the promotion of cardiovascular molecular imaging by holding an invitational meeting at Lake Tahoe in July 2002 and coordinating the first Cardiovascular Molecular Imaging Symposium in 2004 in collaboration with the Society of Nuclear Medicine (SNM). This 2004 invitational meeting brought together basic scientists and imaging scientists for a series of interactive sessions that discussed an array of topics related to cardiovascular molecular imaging. The primary focus of that symposium was radiotracer-based imaging.

To further advance the development of molecular imaging, the SNM created the Molecular Imaging Center of Excellence, which incorporated members from the society having expertise in radiochemistry, instrumentation, and advanced imaging, as well as clinical focuses in oncology, neurology, and cardiovascular medicine. To specifically advance cardiovascular molecular imaging, joint sessions focusing on cardiovascular molecular imaging were held at the annual meetings of multiple scientific societies, including those of the SNM, American Society of Nuclear Cardiology, American College of Cardiology, and American Heart Association. In April and May 2009, with the support of the National Institutes of Health and industry, the Molecular Imaging Center of Excellence in collaboration with several cardiovascular imaging societies coordinated a follow-up symposium, "Multimodality Cardiovascular Molecular Imaging," 
at the National Institutes of Health to advance the field of cardiovascular molecular imaging. This symposium brought together leading investigators from several imaging societies engaged in molecular and cardiovascular imaging, including the SNM, Radiologic Society of North America, American Society of Nuclear Cardiology, Academy of Molecular Imaging, Society of Cardiovascular MRI, and American Society of Echocardiography. This was the first large, multidisciplinary symposium with an expanded focus on multimodality cardiovascular molecular imaging. The current supplement to the journal summarizes the presentations and discussions associated with this landmark symposium.

\section{SYMPOSIUM ON MULTIMODALITY CARDIOVASCULAR MOLECULAR IMAGING}

The 2-day symposium on Multimodality Cardiovascular Molecular Imaging brought together individuals from a wide range of scientific disciplines, including chemistry, engineering, physics, molecular biology, cardiovascular physiology, and imaging sciences, with the goal of promoting the nascent field of cardiovascular molecular imaging. The meeting successfully attracted individuals from both the basic-science and the clinical communities and facilitated interaction and discussion among these diverse communities. The meeting focused on advances in targeted multimodality imaging of the cardiovascular system and included discussions of the imaging of cardiovascular receptors, stem cell therapy, vascular biology, myocardial metabolism, and other biologic processes relevant to the cardiovascular system. The meeting included both invited expert lecturers and original-abstract poster presenters. Support was provided for young investigators to attend the meeting to promote the growth and development of the field. The proceedings of the symposium were posted on the Web site of the Molecular Imaging Center of Excellence (1), and abstracts from the poster session were published in The Journal of Nuclear Medicine (2).

The symposium successfully met several key objectives: to educate a broad scientific community about the potential of targeted multimodality cardiovascular molecular imaging; to provide an overview of critical issues related to the development of targeted labeled tracers; to provide an overview of various technologies for both small-animal imaging and clinical molecular imaging; to review the approaches and implications for imaging of stem cells; to define the potential of imaging of cardiovascular receptors and metabolism; to provide an overview of the potential of molecular imaging for improving our understanding and management of critical cardiovascular pathophysiologic processes, such as atherosclerosis, angiogenesis, cardiomyopathies, ischemia, and infarction; to promote basic cardiovascular molecular imaging research and clinical applications; to foster collaborations between scientists working in various related fields; and to stimulate interaction between junior scientists and more established investigators in the field.
The review articles included in this supplement not only present the state of the art of cardiovascular molecular imaging but also discuss opportunities and challenges the community will face in advancing cardiovascular molecular imaging to clinical practice. Several opportunities are highlighted: the availability of metabolic and receptor imaging for preclinical and clinical applications in the cardiovascular system; the discovery of novel multifunctional targeted probes; new intravascular imaging devices; and new hybrid imaging systems with improved sensitivity and resolution for preclinical imaging. Several challenges in the translation of molecular imaging to practice are also highlighted: equipment designed primarily for noncardiac imaging that has suboptimal sensitivity for imaging small vascular targets and inadequate correction capabilities for cardiac and respiratory motion; the lack of commercially available quantitative software for analysis of dynamic images or targeted hot-spot images of critical molecular or cellular targets; the fact that most cardiovascular imaging is being performed primarily in outpatient cardiology practices with only basic SPECT capabilities; and the limited availability of targeted probes.

\section{SUMMARY}

With health care reform, new challenges come to both the basic-research and the clinical-practice communitieschallenges that will require technologic adaptations and integration into new diagnostic and therapeutic paradigms. Traditionally, cardiovascular disease or therapeutic interventions were detected, evaluated, and prognosticated through the study of late anatomic changes or the physiologic consequences expressed by changes in flow, metabolism, and function. The development of biologically targeted markers reflective of the genetic and cellular processes of disease has become possible with advances in genomics and proteomics. The use of these molecular targets for imaging in preclinical models and the translation of these approaches to patients have become possible with the advent of several technologic advances reviewed in this supplement. The application of molecular imaging may also provide additional unique molecular and pathophysiologic insight that will allow a more personalized approach to evaluation and management of cardiovascular disease.

As interest in multimodality cardiovascular molecular imaging gains momentum, we can expect to see increasing numbers of imaging biomarkers in preclinical and clinical studies. These noninvasive targeted approaches will have to be carefully evaluated for their prognostic value, cost-effectiveness, and potential long-term benefit or toxicity in order to translate these technologic advances into improved patient care.

\section{REFERENCES}

1. Symposium on Multimodality Cardiovascular Molecular Imaging, April 30-May 1, 2009. Available at: http://www.molecularimagingcenter.org/index.cfm?Page ID $=8160 \&$ RPID $=6829$. Accessed Mar. 31, 2010.

2. Poster abstracts from the Multimodality Cardiovascular Molecular Imaging Symposium. J Nucl Med. 2009;50:656-665. 